\title{
DIGITAL FINITE IMPULSE RESPONSE NOTCH FILTER WITH NON-ZERO INITIAL CONDITIONS, BASED ON AN INFINITE IMPULSE RESPONSE PROTOTYPE FILTER
}

\author{
Sławomir Kocoń, Jacek Piskorowski \\ West Pomeranian University of Technology, Faculty of Electrical Engineering, Sikorskiego 37, 70-313 Szczecin, Poland, \\ ( $₫$ slawomir.kocon@zut.edu.pl, +48 91449 5236, jacek.piskorowski@zut.edu.pl)
}

\begin{abstract}
In this paper a concept of finite impulse response (FIR) narrow band-stop (notch) filter with non-zero initial conditions, based on infinite impulse response (IIR) prototype filter, is proposed. The filter described in this paper is used to suppress power line noise from ECG signals. In order to reduce the transient response of the proposed FIR notch filter, optimal initial conditions for the filter have been determined. The algorithm for finding the length of the initial conditions vector is presented. The proposed values of the length of initial conditions vector, for several ECG signals and interfering frequencies, are calculated. The proposed filters are tested using various ECG signals. Computer simulations demonstrate that the proposed FIR filters outperform traditional FIR filters with initial conditions set to zero.
\end{abstract}

Keywords: measurement, signal processing, finite impulse response (FIR) filter, non-zero initial conditions, transient response, electrocardiography.

(C) 2012 Polish Academy of Sciences. All rights reserved

\section{Introduction}

Practical design and implementation of FIR filters have become an important part in the development of measurement, especially in automatic catchweighing instruments [1-2], biomedical devices [3] or communication [4]. The notch filter is a narrow band-stop filter that can be used in various applications for removing noise at known frequencies. Notch filters allow significant reduction of power line interference from measured signals [5]. Unconditional stability and linear phase response are among the advantages of FIR filters [6]. Design methods of FIR notch filters are well known and widely described in literature [6-9]. In the works devoted to the FIR filters authors mainly focus on analysing the frequency response. Properties of these filters in the time domain are rarely discussed in the literature.

In many measurements there is a need to work out the output signal without delay. The transient response of a filter is a secondary effect of the approximation of the frequency response. It may significantly corrupt the initial portion of the processed signal [10] which may be important, e.g. in the Automated External Defibrillators (AED). In this kind of devices it is very important to work out a clean ECG signal as fast and as accurately as possible [11]. AEDs are portable defibrillators that are designed for untrained non-medical personnel. Audible and visual prompts will guide the user through the process. The research papers have shown that the delay from cardiac arrest to delivery of the defibrillation is the most important factor of patient's survival. Successful defibrillation declines at a rate of 7 to $10 \%$ with each minute of delay [11].

A filtered output signal has two components: a transient response and a steady state response. The measurement of the filtered output signal can be accelerated by reducing the 
transient response. The improvement of the transient response is a problem which has been considered in many fields of engineering. However, the commonly used filters are, in many cases, inappropriate due to the long duration of transients [12-13].

For filters with time-invariant coefficients there are only small possibilities of shortening the transient state. In this paper a technique for improving the transient response of the timeinvariant FIR filters using non-zero initial conditions for the filter is proposed. To calculate optimal initial conditions for the filter the projection technique is used [14].

There are many methods that can be used to design FIR notch filters. One of these methods uses the IIR prototype filter [3]. This method will be used in this paper.

This paper is divided into the following sections: Section 2 describes the method of designing a FIR notch filter using the IIR prototype filter. In section 3, the method for calculating the initial conditions for the FIR filter is derived. Then, in Section 4, the analysis of the length of the initial conditions vector is presented. In Section 5, the results of simulation are given. Finally, concluding remarks are drawn in Section 6.

\section{FIR notch filter based on IIR prototype filter}

The filter considered in this paper is based on the notch filter proposed by Deshpande et al [3]. The transfer function of the second order IIR notch filter has the following form:

$$
H_{0}(\mathrm{z})=K_{0} \frac{1-2 \cos \left(\omega_{0}\right) z^{-1}+z^{-2}}{1-2 r \cos \left(\omega_{0}\right) z^{-1}+r^{z} z^{-2}}
$$

where $K_{0}$ is a scaling factor, $r$ is the pole radius, and $\omega_{0}$ is the normalised frequency in radians.

The notch effect in (1) is obtained by placing a pair of complex conjugate zeros at $e^{ \pm j \omega_{0}}$. The frequency response of (1) in the passband is close to unity. This is obtained by placing a pair of conjugate poles at $r e^{ \pm j \omega_{0}}$. The value of $r$ must be positive and smaller than unity.

Equation (1) can be written in the following form [3]:

where:

$$
H_{0}(z)=K_{0} H_{\mathrm{N}}(z) H_{\mathrm{D}}(z),
$$

$$
\begin{gathered}
H_{\mathrm{N}}(z)=1-2 \cos \left(\omega_{0}\right) z^{-1}+z^{-2}, \\
H_{\mathrm{D}}(z)=\frac{1}{1-2 r \cos \left(\omega_{0}\right) z^{-1}+r^{2} z^{-2}} .
\end{gathered}
$$

Since $z=e^{j \omega}$, (2b) can be written as:

$$
\begin{aligned}
H_{\mathrm{N}}\left(e^{j \omega}\right) & =1-2 \cos \left(\omega_{0}\right) e^{-j \omega}+e^{-j 2 \omega}= \\
& =e^{-j 2 \omega}\left(e^{j \omega}-e^{j \omega_{0}}\right)\left(e^{j \omega}-e^{-j \omega_{0}}\right) .
\end{aligned}
$$

If we put $\omega=\omega_{0}$ in (3), it is easy to show that $H_{\mathrm{N}}\left(e^{j \omega_{0}}\right)=0$. This ensures a notch at $\omega_{0}$.

Using long division of unity by factor $\left(1-2 r \cos \left(\omega_{0}\right) z^{-1}+r^{2} z^{-2}\right)$, (2c) can be written as follows:

$$
\begin{aligned}
H_{\mathrm{D}}(z) & =1+a z^{-1}+\left(a^{2}-b\right) z^{-2}+\left(a^{3}-2 a b\right) z^{-3}+ \\
& +\left(a^{4}-3 a^{2} b+b^{2}\right) z^{-4}+\left(a^{5}-4 a^{3} b+3 a b^{2}\right) z^{-5}+\cdots
\end{aligned}
$$

where: $a=2 r \cos \left(\omega_{0}\right)$ and $b=r^{2}$.

Equation (4) can be also put in the following form:

where:

$$
H_{\mathrm{D}}(z)=\sum_{i=0}^{\infty} h_{2}(i) z^{-i}
$$

$$
h_{2}(i)=\sum_{m=0}^{\lfloor x\rfloor}(-1)^{m}\left(\begin{array}{c}
i-m \\
m
\end{array}\right) a^{(i-2 m)} b^{m} .
$$


In (5b), notation $\lfloor x\rfloor$ stands for the integral part of $\mathrm{x}$. According to [3], truncating the series given by (5a) at $i=M$, the FIR filter can be described by the following transfer function:

$$
N_{0}(z)=\sum_{i=0}^{M} D(i) z^{-i}
$$

where:

$$
\begin{gathered}
D(i)=h_{2}(i)-2 \cos \left(\omega_{0}\right) h_{2}(i-1)+h_{2}(i-2), \quad i=2,3, \ldots, L, \\
D(0)=h_{2}(0), \quad D(1)=-2 \cos \left(\omega_{0}\right) h_{2}(0)+h_{2}(1) .
\end{gathered}
$$

It should be noted that the method proposed in this section has some limitations. If the pole radius of the IIR prototype filter is major than 0.93 , ripples in the passband of the obtained FIR filter can be observed. These ripples are caused by round-off noise. The round-off noise is proportional to $1 /(1-r)^{2}$ [7].

Figure 1 presents the comparison of the magnitude responses of the second order IIR notch prototype filter (with pole radius $r=0.93$ and $1.8 \mathrm{rad} / \mathrm{s}$ notch frequency) and the 170th order FIR filter based on this prototype. For the assumed case the maximal error between the magnitude responses of the designed FIR notch filter and the IIR prototype filter over the entire frequency range is equal to 0.0251 .

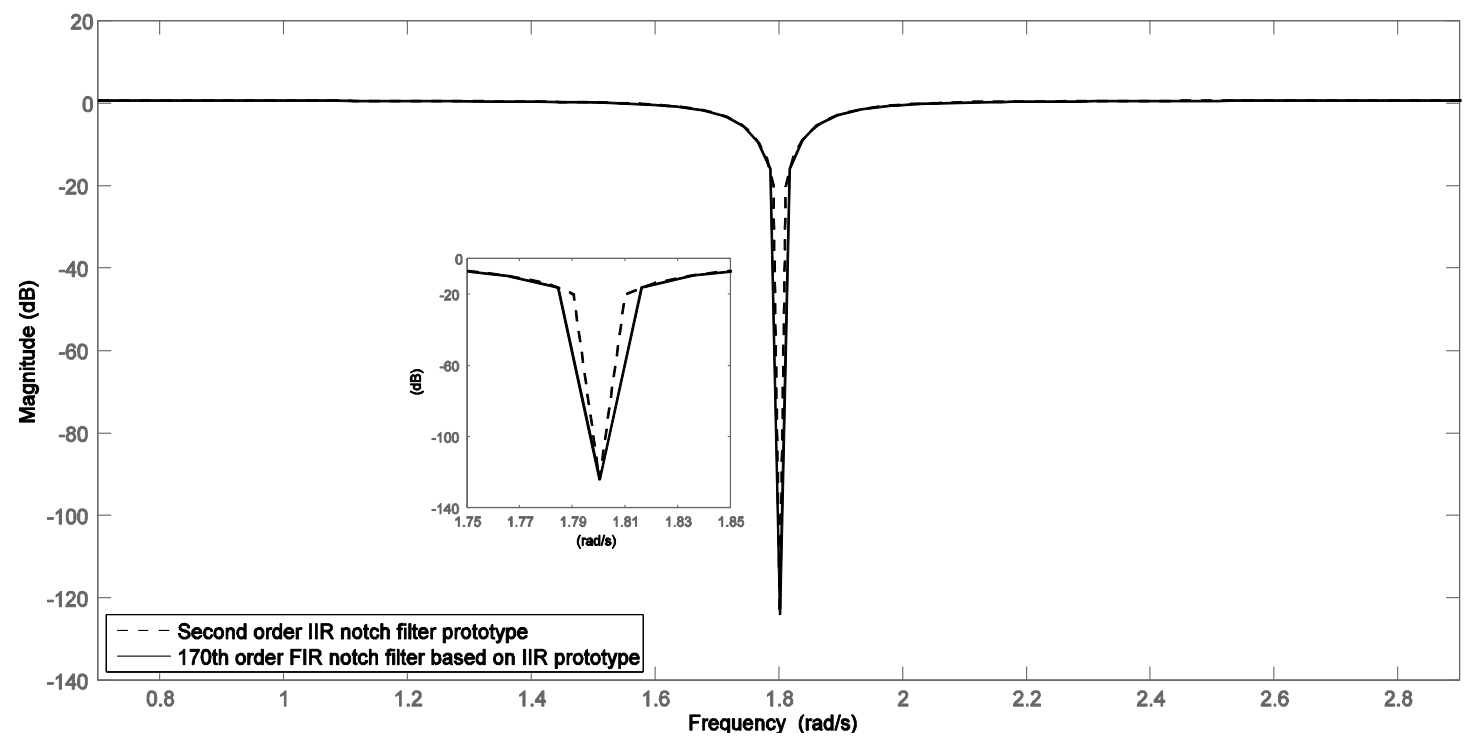

Fig. 1. Comparison between the magnitude responses of the IIR notch prototype filter and the designed FIR filter.

\section{Non-zero initial condition for the FIR filter}

An FIR filter based on the IIR prototype filter has many advantages, e.g. unconditional stability [6]. Conversely, the transient response of the IIR prototype filter also occurs in the output of the designed FIR filter [3]. The transient response duration strongly depends on the rejection bandwidth [10]. Usually, if the rejection bandwidth is narrower, a longer transient response is observed. However, to reduce a sinusoidal interference without distortion in the frequency domain, notch filters with very narrow rejection bandwidth are chosen [14]. In traditional design methods of notch filters the trade-off between the duration of the transient response and the distortion of separateness in the frequency domain must be considered [14]. In this paper a technique to suppress the transient response in FIR notch filters based on prototype IIR filters is proposed. 
The transient response suppressing method is based on the vector projection technique [14]. First of all, the vector projection method is used to decompose first $L$ samples of the filter input signal into a sinusoidal component and a useful signal component. Then, the initial values for the filter are calculated. In the next step, first $L$ samples of the filter output signal are replaced by formerly determined initial conditions.

A useful signal distorted by a sinusoidal interference can be written as follows [14]:

$$
x(n)=u(n)+s(n),
$$

where: $x(n)$ is the notch filter input signal, $u(n)$ is the useful signal, and $s(n)$ is the sinusoidal interference with amplitude $A_{0}$, normalised frequency $\omega_{0} \mathrm{in} \mathrm{rad} / \mathrm{Sa}$, and initial phase $\phi$, given by:

$$
s(n)=A_{0} \sin \left(\omega_{0} n+\phi\right) .
$$

The transient response suppression technique is presented as follows [14]. At the beginning, $L$ samples of the signal $x(n)$ are put into vector $\boldsymbol{X}$ :

$$
\boldsymbol{X}=\left[\begin{array}{c}
x(0) \\
x(1) \\
\vdots \\
x(L-1)
\end{array}\right]
$$

Using (7) and (9), vector $\boldsymbol{X}$ can be rewritten as:

$$
\boldsymbol{X}=\boldsymbol{U}+\boldsymbol{S}
$$

where:

and

$$
\boldsymbol{U}=\left[\begin{array}{c}
u(0) \\
u(1) \\
\vdots \\
u(L-1)
\end{array}\right]
$$

$$
\boldsymbol{S}=\left[\begin{array}{c}
s(0) \\
s(1) \\
\vdots \\
s(L-1)
\end{array}\right]
$$

Equation (8) can be written in the following form:

$$
s(n)=A_{0} \sin \left(\omega_{0} n+\phi\right)=A_{0}\left[\cos (\phi) \sin \left(\omega_{0} n\right)+\sin (\phi) \cos \left(\omega_{0} n\right)\right] .
$$

Using (11), matrix $\boldsymbol{A}$ can be defined as follows:

$$
\boldsymbol{A}=\left[\begin{array}{cc}
1 & 0 \\
\cos \left(\omega_{0}\right) & \sin \left(\omega_{0}\right) \\
\cos \left(2 \omega_{0}\right) & \sin \left(2 \omega_{0}\right) \\
\vdots & \vdots \\
\cos \left[(L-1) \omega_{0}\right] & \sin \left[(L-1) \omega_{0}\right]
\end{array}\right]
$$

In matrix (12) vector $\boldsymbol{S}$ is in the row space for any initial phase $\phi$ of $s(n)$.

The next step of the projection method is to define the projection operator $\boldsymbol{P}$. This operator is defined as:

$$
\boldsymbol{P}=\boldsymbol{A}\left(\boldsymbol{A}^{T} \boldsymbol{A}\right)^{-1} \boldsymbol{A}^{T},
$$

where notation $T$ stands for the transposition. Using matrices (12) and (13) the useful signal can be estimated by: 


$$
\overline{\boldsymbol{U}}=(\boldsymbol{I}-\boldsymbol{P}) \boldsymbol{X},
$$

where $\boldsymbol{I}$ is the identity matrix. The sinusoidal interference can be estimated by:

$$
\overline{\boldsymbol{S}}=\boldsymbol{P} X .
$$

Using the estimated useful signal $\overline{\boldsymbol{U}}$ as initial condition for the FIR notch filter, the transient response can be considerably reduced. The output of the $N$-th order FIR filter is given by [4]:

$$
y(n)=\left[b_{0}(n)+b_{1}(n-1)+b_{2}(n-2)+\cdots+b_{N}(n-N)\right] x(n),
$$

where: $b_{0}(n), b_{1}(n-1), b_{2}(n-2), \ldots, b_{N}(n-N)$ are the FIR filter coefficients, $y(n)$ and $x(n)$ are the filter output and input, respectively. For $n=0$ to $L-1$, values of the filter output $y(n)$ are replaced by the estimated signal $\overline{\boldsymbol{U}}$ :

$$
y(n)=[y(0) y(1) \ldots y(L-1)]^{T}=\overline{\boldsymbol{U}}=(\boldsymbol{I}-\boldsymbol{P}) \boldsymbol{X} .
$$

\section{Length of non-zero initial condition vector}

In this section the optimization process of the length of the non-zero initial conditions vector for the proposed filter is presented. The main problem of using the technique described in the previous section rests upon proper selection of $L$ (the length of the initial conditions vector). The authors in [13] denoted that the value of $L$ should be integer between 5 and 15 . A proof of that statement is provided. In this paper the analysis of the length of the initial conditions vector is done. In order to investigate the dependence of the quality of filtering and length of the initial conditions vector, the ECG signal distorted by $50-\mathrm{Hz}$ power line interference is used. The distorted ECG signal used in this paper is presented in Fig. 2. The signal was by a $50-\mathrm{Hz}$ sinusoid with unity amplitude. The signal is sampled at $f_{s}=250 \mathrm{~Hz}$.

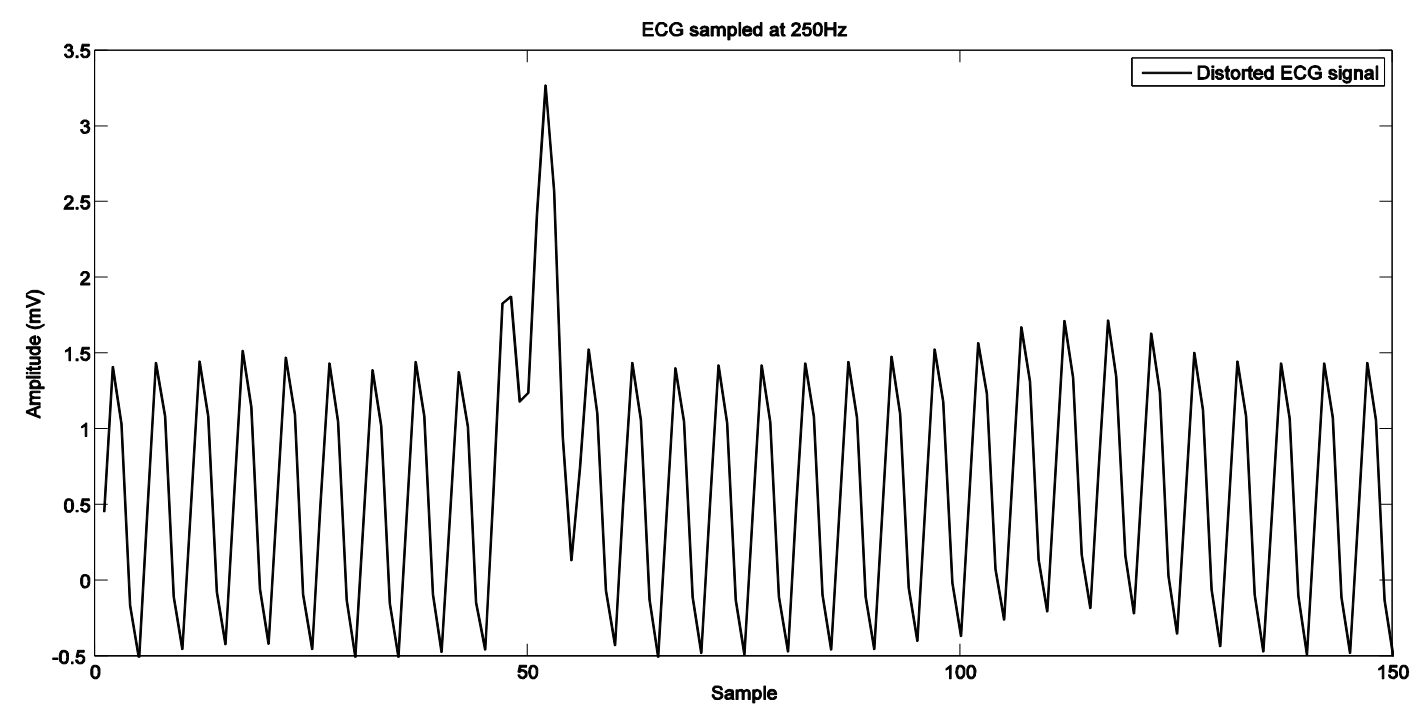

Fig. 2. ECG signal with 50-Hz distortion.

In Fig. 3, the mean squared error (MSE) is shown as a function of initial condition vector length. The MSE is defined as:

$$
E=\frac{1}{K} \sum_{n=1}^{K}|y(n)-x(n)|^{2},
$$


where $K$ is equal to $90, y(n)$ is the FIR filter output, and $x(n)$ is the FIR filter input signal. The MSE of the filter, with values of $L$ from 1 to 45, was calculated. The optimal value of $L$ can be easily picked out using this figure.

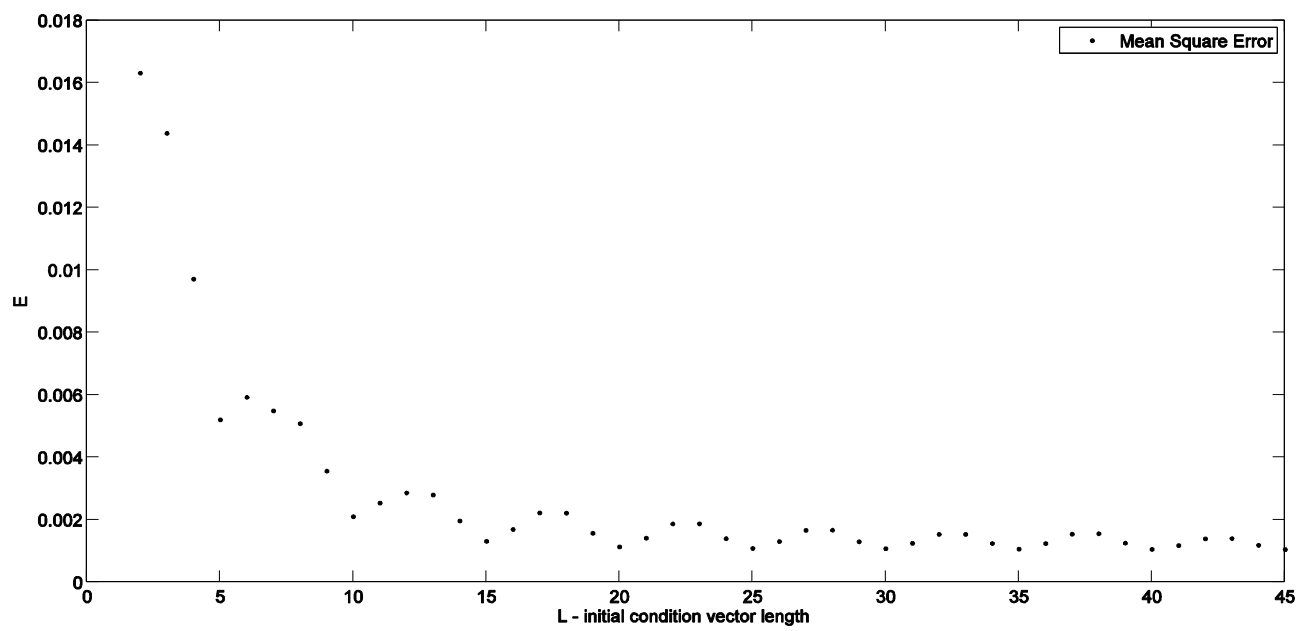

Fig. 3. Mean squared error as a function of initial condition vector length.

As it can be seen in Fig. 3 the value of $L$ proposed in [13] does not provide the minimal value of the MSE. The local minima ${ }^{*} L$ of the MSE are correlated with the sampling rate $f_{s}$ and noise frequency $f_{0}$ as follows:

$$
{ }^{*} L=\frac{f_{s}}{f_{0}} k, \quad k=1,2,3, \ldots \mathrm{s} .
$$

For the used ECG signal, the local minima of the MSE, presented in Fig. 3 appear when $L$ is equal to $\frac{250}{50} k=5 k, k=1,2,3, \ldots$

In the case of improper choice of the value of $L$, ripples in the estimated useful signal $\overline{\boldsymbol{U}}$ occur. In the technique used to suppress the transients, the first $L$ samples of the filter output signal are replaced by the estimated useful signal $\overline{\boldsymbol{U}}$. To avoid problems with computational complexity and delays of the output signals, the minimum value of $\mathrm{L}$ that ensures the smallest value of the MSE should be selected. Therefore, a properly chosen value of $L$ guarantees good quality of filtering and suppression of the transient response. If we define transient response duration in samples by $M$, the value of $L$ should be the first ${ }^{*} \boldsymbol{L}$ from (18) greater than $M$. Proper selection of the value of $L$ provides a significant reduction of transient response, because all samples of transient response are replaced by $\overline{\boldsymbol{U}}$.

In Table 1 the proposed values of $L$ calculated using (18) are presented. In the next section simulations using the proposed values of $L$ are carried out.

Table 1. The proposed value of the initial condition vector length $L$.

\begin{tabular}{|l|c|c|c|c|c|}
\hline $\begin{array}{c}\text { Sample rate } \\
f s[\mathrm{~Hz}]\end{array}$ & $\begin{array}{c}\text { Noise frequency } \\
f_{0}[\mathrm{~Hz}]\end{array}$ & $f s / f_{0}$ & $\begin{array}{c}\text { Transient } \\
\text { response } \\
\text { duration } M\end{array}$ & $k$ & $\begin{array}{c}\text { The proposed } \\
\text { value of } L\end{array}$ \\
\hline \multirow{2}{*}{250} & 50 & 5 & 30 & 6 & 30 \\
\hline \multirow{2}{*}{360} & 60 & $4.16(6)$ & 33 & 8 & 33 \\
\cline { 2 - 6 } & 50 & 4.2 & 28 & 5 & 36 \\
\hline \multirow{2}{*}{1000} & 60 & 6 & 28 & 5 & 30 \\
\cline { 2 - 6 } & 50 & 20 & 41 & 3 & 40 \\
\hline
\end{tabular}




\section{The results of simulations}

Simulations of the proposed FIR filter with non-zero initial conditions have been made using Mathworks Matlab R2011b. For examination, three different ECG signals have been used. The first (ECG1) and the second (ECG2) signal is the voltage between the left leg electrode (LL) and the right arm electrode (RA). The third signal (ECG3) is taken from electrode (V1) placed in the fourth intercostal space just to the right of the sternum. The first signal is unconditioned, the second and the third are conditioned, amplified by 200 analog to digital unit per miliVolt (adu/mV).

The clean ECG signal contains frequency components ranging from $0.05 \mathrm{~Hz}$ to $100 \mathrm{~Hz}$. Therefore, the 50-Hz interference, which has to be removed from the useful signal, lies inside the spectrum of the ECG signal. ECG1 is sampled at $250 \mathrm{~Hz}$ but ECG2 and ECG3 are sampled at $360 \mathrm{~Hz}$. All the ECG signals originate from the MIT-BIH (Massachusetts Institute of Technology-Beth Israel Hospital). In this section, first of all, the influence of the value of $L$ on the filtration process is investigated. To evaluate the performance of the proposed FIR filter the MSE given by (17) is calculated. Secondly, simulations of the discussed filter are carried out. Simulations are made with three ECG signals starting at different points, i.e. at QRS complex or PR interval. The MSEs for each case are calculated. Also the MSEs for the second order IIR notch filter are determined.

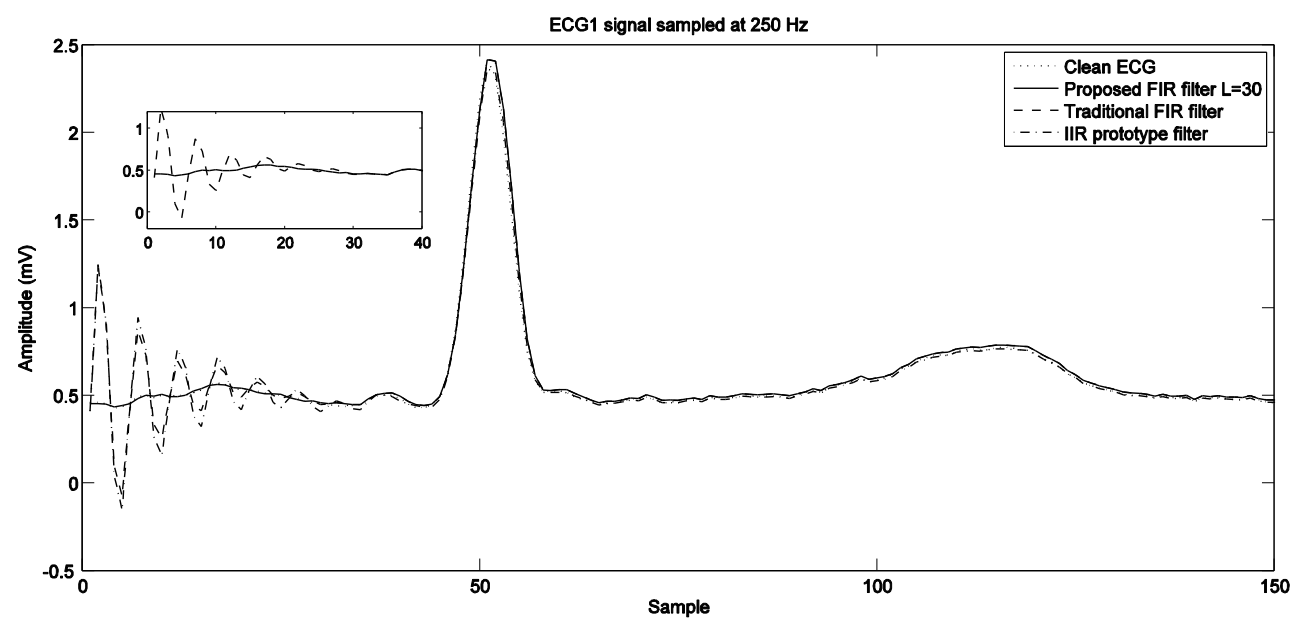

Fig. 4. Filtering results. The value of $\mathrm{L}$ is appropriate.

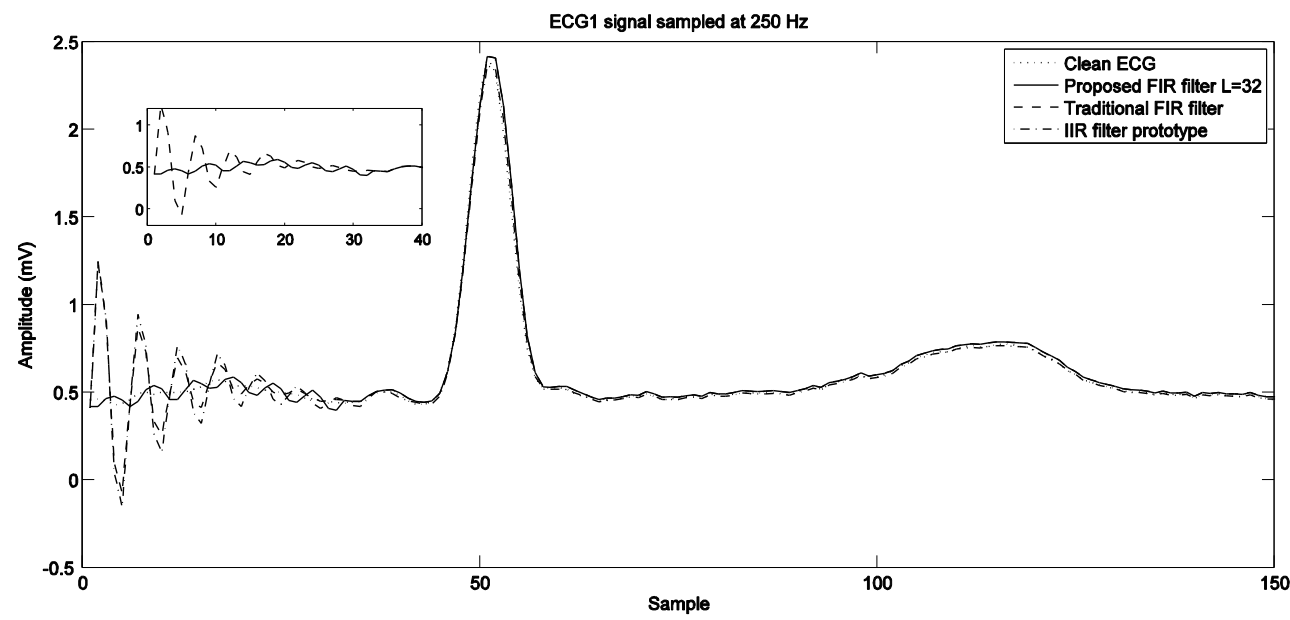

Fig. 5. Filtering results. The value of $\mathrm{L}$ is inappropriate. 
In Figs. 4 and 5 the simulations of filters with proper and improper choice of the value of $L$ are presented. Proper selection of $L$ assures suppression of the transient response. On the other hand, an incorrect value of $L$, presented in Fig. 5, causes unintentional ripple in the filter output signal. Proper values of $L$ are presented in Table 1 . The MSEs in the discussed cases are 0.001077 and 0.001541 respectively. The MSE for the second order IIR notch filter is 2.057016 .

The next simulations provide the results of the various ECGs filtering processes. The ECG signal in each simulation begins at different moments. It is extremely important in the ECG measurement to have a clean signal as quickly as possible. The portion of the ECG signal lost on the transient response may impede appropriate measures. In the cases assumed below, according to (18), a minimum value of the MSE occurs when $L$ is an integer greater than the transient response duration $M$. For ECG2 and ECG3 signals it was assumed that $\boldsymbol{f}_{\boldsymbol{s}}=$ $360 \mathrm{~Hz}, \boldsymbol{f}_{\mathbf{0}}=\mathbf{5 0 ~ H z}$, and $M=28$. According to Table $1, L$ should be set to $\mathbf{3 6}$.

In Figs. 6 and 7 the output signals of the proposed FIR filters are presented. The input of the filters are ECG2 and ECG3, both with $50-\mathrm{Hz}$ distortion. The simulations start at the PR interval (flat part of the ECG) and the QRS complex (peak of the ECG), respectively.

a)

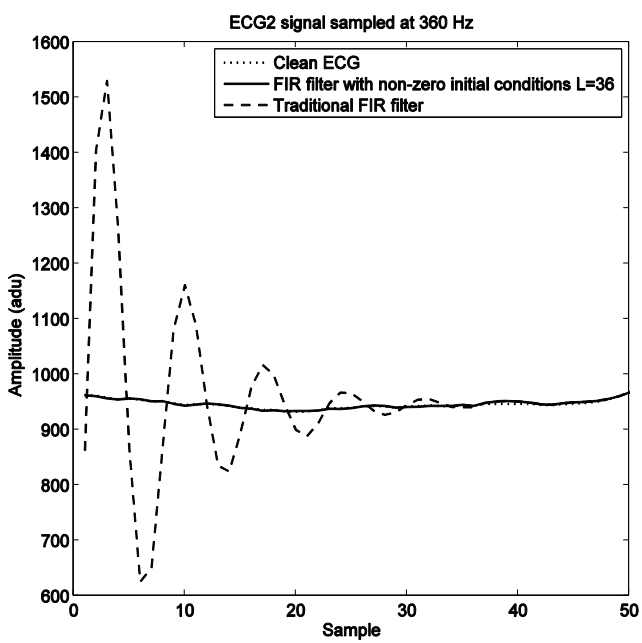

b)

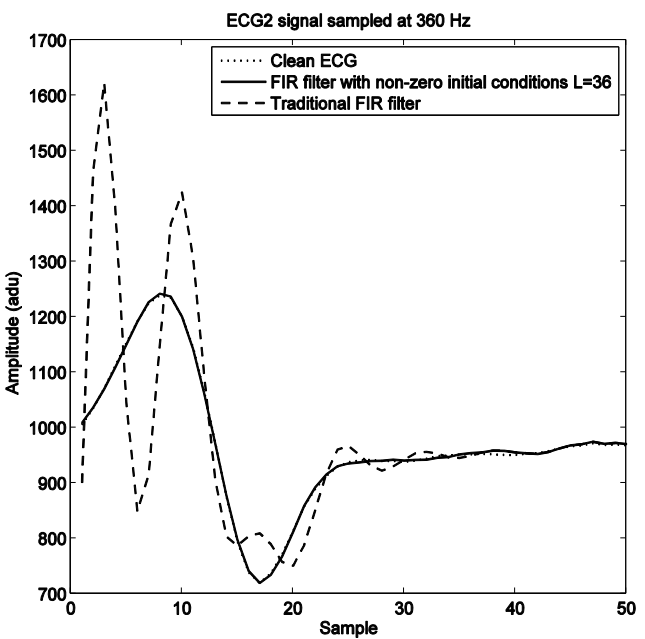

Fig. 6. ECG2 filtering results: a) simulation start at PR interval; b) simulation start at QRS complex.

a)

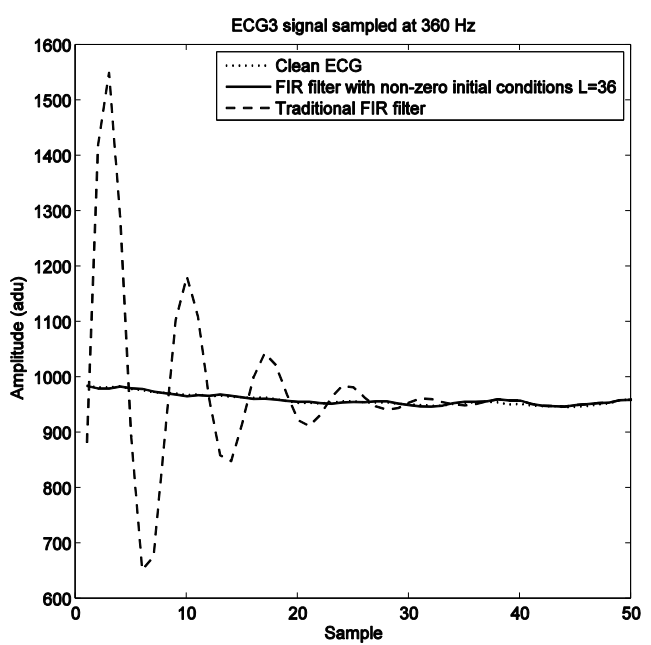

b)

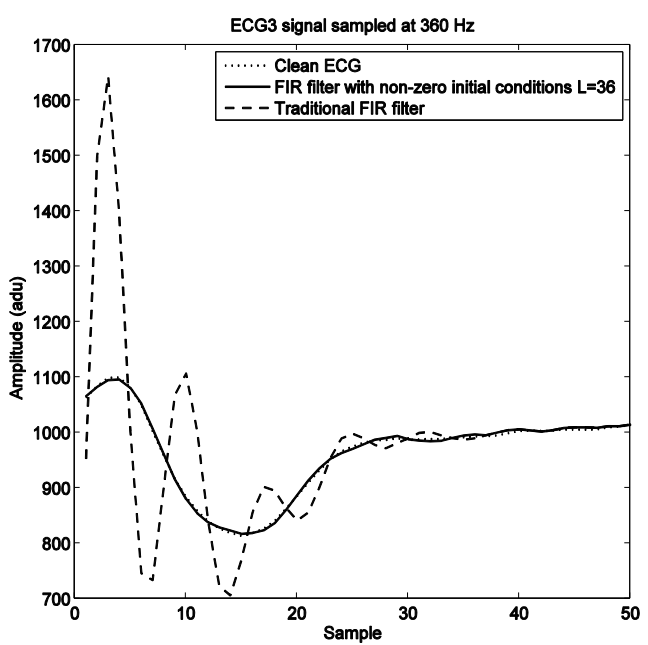

Fig. 7. ECG3 filtering results: a) simulation start at PR interval; b) simulation start at QRS complex. 
As shown in Figs. 6 and 7, the described FIR filter with non-zero initial conditions outperforms the traditional FIR filter in any case. Using the proposed FIR filters in the ECG measurements the transient response and 50-Hz distortion is reduced. In Table 2 the results of simulations are presented. For each signal (ECG1, ECG2, ECG3) four simulations have been made. The first two started at the PR interval, the last two at the QRS complex of ECG. For both cases the value of $L$ is selected in a proper and improper manner, respectively. The MSE is calculated using (17).

Table 2. Results of numerical experiments for different input signals.

\begin{tabular}{|l|c|c|c|}
\hline \multicolumn{1}{|c|}{ The FIR filter input signal } & $\begin{array}{c}\text { MSE } \\
\text { Traditional } \\
\text { FIR filter }\end{array}$ & $\begin{array}{c}\text { MSE } \\
\text { Proposed } \\
\text { FIR filter }\end{array}$ & $\begin{array}{c}\text { MSE } \\
\text { IIR filter }\end{array}$ \\
\hline ECG1, $L=30$, starts at PR interval & 0.01839 & 0.00108 & 0.01838 \\
\hline ECG1, $L=33$, starts at PR interval & 0.01839 & 0.00154 & 0.01838 \\
\hline ECG1, $L=30$, starts at QRS complex & 0.01322 & 0.00040 & 0.01338 \\
\hline ECG1, $L=33$, starts at QRS complex & 0.01322 & 0.00114 & 0.01338 \\
\hline ECG2, $L=36$, starts at PR interval & 11003.5 & 133.987 & 11003.6 \\
\hline ECG2, $L=32$, starts at PR interval & 11003.5 & 3491.88 & 11003.6 \\
\hline ECG2, $L=36$, starts at QRS complex & 10527.1 & 5.73273 & 10527.1 \\
\hline ECG2, $L=32$, starts at QRS complex & 10527.1 & 3295.92 & 10527.1 \\
\hline ECG3, $L=36$, starts at PR interval & 10624.0 & 38.4847 & 10624.0 \\
\hline ECG3, $L=32$, starts at PR interval & 10624.0 & 3528.00 & 10624.0 \\
\hline ECG3, $L=36$, starts at QRS complex & 9759.28 & 6.60793 & 9759.29 \\
\hline ECG3, $L=32$, starts at QRS complex & 9759.28 & 3760.80 & 9759.29 \\
\hline
\end{tabular}

For all input signals, it is obvious that the FIR filter with non-zero initial conditions has always smaller MSE than the traditional FIR filter with initial conditions set to zero. The projection operator matrix $\boldsymbol{P}$ is dependent only on $L$ and $\boldsymbol{\omega}_{\mathbf{0}}$. The matrix $(\boldsymbol{I}-\boldsymbol{P})$ could be pre-stored for various classes of signals [14]. The presented technique for reducing the transient response is easy to implement to FIR filters and provides smaller MSE that the traditional FIR filters. The implementation cost for FIR filter structures is larger than for IIR filters discussed in [10]. One of the important advantages of FIR filters is their unconditional stability.

\section{Conclusions}

In order to reduce the transient response of the FIR notch filter, non-zero initial conditions have been proposed. To compute them, the vector projection method has been used. The analysis of influence of the number of non-zero initial conditions on filtering quality has been carried out. The optimization process of the initial conditions vector length has been presented. The proposed FIR notch filter has been successfully used to reduce the transient response of signals distorted by $50-\mathrm{Hz}$ sinusoidal interference. Simulation results prove, for various ECG signals, that this technique can improve the performance of FIR notch filters based on an IIR prototype filter. Furthermore, the values of initial conditions vector length for several sampling rates and interference frequencies have been presented. 


\section{Acknowledgements}

This work has been supported by the Ministry of Science and Higher Education of the Republic of Poland under grant contract N N505 484740.

\section{References}

[1] Pietrzak, P. (2009). Fast filtration method for static automatic catchweighing instruments using a nonstationary filter. Metrol. Meas. Syst., 14(4), 669-676.

[2] Tasaki, R., Yamazaki, T., Ohnishi, H., Kobayashi, M., Kurosu S. (2007). Continuous weighing on a multistage conveyor belt with FIR filter. Measurement, 40(7-8), 791-796.

[3] Deshpande, R., Kumar, B., Jain, S.B. (2012). On the design of multi notch filters. International Journal of Circuit Theory and Applications, 40(4), 313-327.

[4] Hjorungnes, A. (2005). Minimum symbol error rate transmitter and receiver FIR MIMO filters for multilevel PSK signalling. In Proc. of ISWCS 2005, Siena, Italy, 27-31.

[5] Piskorowski, J. (2010). Digital notch filter with time-varying quality factor for the reduction of powerline interference. In Proc. of IEEE ISCAS, Paris, France, 2706-2709.

[6] Oppenheim, A.V., Schafer, R.W., Buck, J.R. (1999). Discrete-Time Signal Processing. Englewood Cliffs, NJ, Prentice-Hall.

[7] Dutta Roy, S.C., Jain, S.B., Kumar, B. (1994). Design of digital FIR notch filters. IEE Proc. Vision, Image and Signal Processing, 141(5), 334-338.

[8] Zahradnik, P., Vlcek, M. (2004). Fast analytical design algorithms for FIR notch filters. IEEE Transactions on Circuits and Systems-I., Regular Papers, 51(3), 608-623.

[9] Słowik, A. (2011). Application of evolutionary algorithm to design minimal phase digital filters with nonstandard amplitude characteristics and finite bit word length. Bull. Pol. Acad. Sci.-Tech. Sci., 59(2), 125135.

[10] Piskorowski, J. (2010). Digital Q-varying notch IIR filter with transient suppression. IEEE Trans. on Instrumentation and Measurement, 59(4), 866-872.

[11] Lim, W.L., Hang, C.C., Neo, K.B. (2008). Discontinuous Innovations Framework: A Review of Automatic External Defibrillators in the Healthcare Industry. In Proc. of IEEE ICMIT, Bangkok, Thailand, 356-361.

[12] Piskorowski, J., Kaszyński, R. (2001). Analytical synthesis of parameter-varying filter of constant component with application to switching systems. Metrol. Meas. Syst., 18(3), 471-480.

[13] Kaszyński, R. (1998). The properties of parametric filter of signal constant component. Metrol. Meas. Syst., 5(4), 303-313.

[14] Pei, S.-C., Tseng, C.-C. (1995). Elimination of AC interference in electrocardiogram using IIR notch filter with transient suppression. IEEE Trans. on Biomedical Engineering, 42(11), 1128-1132. 